Menge enthalten. Dahingegen war kein Stärkezucker zum sauren weinsauren Ammoniak gedrungen.

Versuche, reinen Rohrzucker auf die Weise in Gahrung zu bringen, wie es mir mit dem Stärkezucker gelungen ist, gaben ein negatives Resultat.

In Wasser suspendirte reine Stärke, auch dünner Kleister aus reiner Stärke bereitet, durch Digeriren oder Kochen mit neutralem oder weinsaurem Ammoniak bemerkenswerth zu verändern, gelang ebenfalls nicht.

Auch reine in Wasser suspendirte Stärke mit erschöpftem Strohpulver versetzt, mit oder ohne Zusatz von saurem weinsaurem Ammoniak, und in Gährungstemperatur gesetzl, veränderte sich nicht.

Es wäre gewiss von Interesse, einige in dieser $\mathbf{A b}$. handlung beschriebene Versuche, statt mit weinsaurem oder citronensaurem Ammoniak, mit Salzen, die flüchtige Pllanzenbasen enthalten (Anilin, Coniin, Nicotin) anzustellen, ferner auch harnsaures und asparaginsaures Ammoniak dazu in Anwendung zu bringen.

\title{
Untersuchungen der im Handel vorkommenden Oblaten; \\ von
}

Witting und Zachau.

1. Weisse Oblaten.

Wir unterwarfen diese desshalb einer Untersuchung, da man in Belgien zum Weissfärben des Brodes $u$. s. w. Bleiweiss (Kremnitzerweiss) und Wismuthoxyd benutzt haben soll. Wir haben jedoch, indem verschiedene Oblaten dieser Art mit

a) Schwefelammonium behandelt, auch

b) Löthrohrversuche nicht unbenutzt gelassen wurden. niemals die Gegenwart jener metallischen Substanzen constatiren können.

Bemerkung. Interessant dürften auch die von Jaquemyes (Annales de Chimie 1831) angestellten Unter- 
suchungen über Ermittelung des schwefelsauren Kupferoxydhydrates im Weissbrode sein, welches um die Farbe mehr zu heben (sowie Smalte beim Papier) dem Teige zugesetzt ward. Schon Kaliumeisencyanürlösung deutete durch einfaches Befeuchten der Masse die Gegenwart des Kupferoxyds an.

\section{Schwarze Oblaten.}

Sie erscheinen nur durch feine animalische Kohle gefärbt. Letztere ward durch Behandlung einer Menge von Oblaten mit siedendem Wasser, nach einigem Stehen der Flüssigkeit, gesondert.

\section{II. Rothe Oblaten.}

Sie waren der wichtigste Gegenstand vorgedachter Oblatenuntersuchungen. Schon durch das Löthrohr zeigte sich auf Kohle bei einer Oblate der eigenthümliche, gelbe Anflug des Bleioxydes im Anfange, dem nachher ein wirkliches Bleikorn folgte; genauer durch die Loupe zu entdecken, jedoch vollkommen sichtbar, wenn mehrere Oblaten zusammen angewandt wurden. Alle übrigen Untersuchungen stimmten dahin überein, dass sie mit Mennige (rothem Bleioxyd) gefärbt waren. In Betreff des quantitativen Verhältnisses fand sich:

a) dass sechs Oblaten, dreissig Gran wiegend, an Bleioxyd $=0,55 \mathrm{Gr}$. enthalten; daher eine Oblate von $\frac{5}{6}$ Gr. $=0,1527$ Gr. des Farbenmaterials, mithin 1 Gr. der Oblate 0,1833 Bleioxyd, = 0,1374 Bleimetall entsprechend, enthält; indem 100 Theile Bleioxyd $=90$ Blei +10 Sauerstoff enthalten. - In Beziehung auf Reaction solcher Substanzen, durch welche gedachtes Bleioxyd in seiner Mehlverbindung nachtheilige Eigenschaften auf den thierischen Organismus ausüben dürte, haben wir besonders Nachfolgendes hervorzuheben.

b) Die Oblaten, mit kaltem Wasser während mehrerer Tage lang macerirt, theilten demselben keine Bleitheile mit, indem die bezeichneten Reagentien, namentlich Schwefelwasserstoffverbindungen, sich durchaus indifferent verhielten.

c) Die mit Wasser benetzten Oblaten zeigten fast 
momentan, indem sie mit jenen Reagentien durch Auftröpfeln in Beruhrung gesetzt wurden, den Bleigehalt an.

d) Die rothen Oblaten jedoch, kurze Zeit mit seh" verdünnter Salpeter - und Salzsäure in Verbindung gesetzt, gaben schon nach einigen Stunden mit Schwefelammonium das Metall zu erkennen.

e) Besonders beobachtungswerth duirfte das Verhalten der Oblaten gegen den Speichel sein. Dieser letztere zeigte folgende Erscheinungen:

a) Er löste rasch, sich gleichsam mit der Oblate coagulirend, dieselbe auf, und eben so schnell erfolgte die Reaction mit den Hydrosulphuren.

b) Schon durch das Befeuchten mit Speichel kann aus einer rothen Oblate die Gegenwart des Bleioxydes nachgewiesen werden, wozu namentlich auch die Wäme, welche Geschmacksorganen eigenthümlich ist, kräftig mitwirkt.

Anmerlung. Es dürfte hier zunächst die Frage aufzustellen sein, ob der Speichel nicht in Betreff seiner Bestandtheile manchen Veränderungen unterworfen ist, herbeigeführt durch verschiedenartige Speisen, Lebensweise u.s.w. Der Speichel, welchen ich benutze, reagirte weder alkalisch noch sauer, und schien nebst phosphorsauren Verbindungen solche von Chlor zu enthalten. Dass aber Salze dieser Art auflösend auf gewisse Metalle wirken, ist bekannt. Wir möchten uibrigens auch hier besonders den Speichelstoff (Ptyalin) hervorhęben.

$$
\text { IV. Gelbe Oblaten. }
$$

Erschienen unsern Untersuchungen zufolge:

a) theilweise durch Curcuma-Aufguss gefärbt. Schon durch Einwirkung des Ammoniakgases zeigte sich die bräunliche Reaction. Löthrohrversuche und andere liessen durchaus keine schädlichen Bestandtheile entdecken.

b) war bei den orangefarbenen Oblaten auch Safranaufguss benutzt worden.

\section{Blaue Oblaten}

fanden sich durch Indigosolution gefärbt. Dass übrigens hier schwefelsaure Indigo-Auflösung benutzt wurde, ergab 
Untersuclungen der im Handel vorkommenden Oblaten. 29

sich durch Behandlung der Oblaten mit heissem Wasser und nachherigem Zusatz von Barytsalzen durch den betreffenden Niederschlag.

\section{Grüne Oblaten.}

Wir fanden dieselben in den meisten Fällen colorirt:

A. durch Pflanzenpigmente, und namentlich hier:

a) Indigo-Auflösung mit Curcuma-Aufguss.

Als Beweis der chemischen Reaction hierher gehöriger Prïfungsmittel erwähnen wir den Einfluss der Aetzalkalien, wodurch das blaue Pigment (Indigo) entfärbt ward, hingegen das gelbe mil einer intensiv braunen Farbe prävalirend zum Vorschein kam.

B. In zwei Fällen fanden sich unverkennbar die Spuren von Kupferoxyd, und hier muthmasslich essigsaures Kupferoxyd (Grünspan), denn es wurden durch verdünnte Salpeter- und Hydrochlorsäure die Oblaten sehr bald entfärbt, und die Solutionen deuteten:

a) durch Schwefelammonium (Niederschlag mit demselben) Kupfer an.

b) So auch Kalıumeisencyaniir.

c) Ammoniak vermittelst Digestion durch die blaue Farbe (schwächere Reaction).

d) Ward die mit verdünnter Salpetersäure angefeuchtete Oblate sehr bald durch Schwefelammonium braun gefärbt.

e) Durchs Löthrohr konnte der Kupfergehalt erst bei Anwendung mehrerer Oblaten ermittelt werden, indem zugleich der Rückstand vorgedachter Untersuchungen unterworfen ward.

f) Zeigte Speichel keine energische Wirkung darauf, nur erst nach längerer Digestion.

Schlussfolge aus obigen Resultaten.

1) Können die rothen Oblaten als schädlich und zwar mit Mennige gefärbt, betrachtet werden; indem den Untersuchungen zufolge schon der Speichel eine Auflösung von Bleioxyd veranlasst. $O b$ nun nicht bei dem häufigen Gebrauche der rothen Oblaten nach und nach bei den Individuen eine förmliche Intoxation erfolgen könne, wenn 
auch nur in einzelnen Partikelchen Bleioxyd den Verdauungswerkzeugen mitgetheilt wird, dürfte jedenfalls als eine medicinal-polizeiliche Aufgabe den Sanitätsbehörden zu empfehlen scin. Das förmliche Verschlucken der rothen Oblaten ist gewiss schädlich, da der Magensaft selbst Antheile von Hydrochlorsäure enthält, und so ctwa gebildetes Chlorblei nachtheilig wirken könnte.

2) Erscheinen die (nach unsern Beobachtungen nur schr wenig im technischen Leben vorkommenden) grünen, mit Kupferoxyd gefärbten Oblaten, sodann nur schädlich, wenn sie verschluckt werden.

3) Dahingegen sind alle übrigen Farben, als von Pflanzenpigmenten entnommen, durchaus unschädlich.

Sollten nun Vorschläge in Betreff unschädlicher Farben gemacht werden, so dürfte dieses nur für die rothe Farbe geschehen, da die ubrigen Farben bereits als Pflanzenpigmente charakterisirt sind ; vergleiche obige Untersuchungen und zwar:

I. weisse Oblaten, ohne Zusatz, durch feines Spelzmehl;

II. schwarze Oblaten, vermittelst feiner animalischer oder vegetabilischer Kohle (Kienruss);

III. gelbe Oblaten, durch Curcuma- oder Safranpigment;

IV. blaue Oblaten, durch Lackmus - und Indigopigment;

V. grüne Oblaten, durch Verbindung der gelben und blauen Pigmente.

VI. rothe Oblaten, für diese als schädlich befundene, duirten vorzugsweise Cochenillefarbstoff, oder auch Fermanbukholz mit Alaunzusatz in Vorschlag zu bringen sein.

\section{Ueber Benutzung deutscher Euphorbia-Arten; von \\ li. Stickel, \\ Apotheker zu Kaltennordheim.}

Nur Weniges können wir in dieser Beziehung über diese so allgemein in Deutschland vorkommenden und stets gedeihenden Pflanzen bis jotzt anführen.

Die sonst gebräuchlichen Radices Euphorbiae Cypa- 